\title{
Harmonic Domain Periodic Steady State Modeling of Power Electronics Apparatus: SVC and TCSC
}

\author{
Leonardo T. G. Lima, Member, IEEE, Adam Semlyen, Life Fellow, IEEE, and M. R. Iravani, Fellow, IEEE
}

\begin{abstract}
The paper presents a general model for the representation of a thyristor-controlled reactor (TCR) in the frequency domain. This model underlies the implementation of a NewtonRaphson procedure to determine the periodic steady state of the power system with fast convergence and high accuracy. The TCR model is used as a building block to represent more sophisticated power electronics apparatus such as the static VAr compensator (SVC) and the thyristor-controlled series capacitor (TCSC).
\end{abstract}

Index Terms-Power electronics, switched systems, thyristor circuits, power system harmonics, harmonic analysis, nonlinear systems.

\section{INTRODUCTION}

A PPLICATIONS of power electronics apparatus in power systems have been spreading and diversifying over the years. This can be asserted by the number of installed units, by the multitude of proposed applications, and by the variety of technologies and structures devised to implement the equipment.

This wide-spread presence requires the accurate representation of such equipment in power system studies and simulations, with a need for detailed models [1]. This requirement is clearly exemplified when different power electronics concepts can be applied to provide the same power system function as, for instance, reactive shunt compensation. The technical justification for using a conventional SVC or a STATCOM should be based on the accurate assessment of the inherent characteristics of these devices and their impact on the system performance.

The need for specific and accurate models is all-important when the periodic steady state is needed, either to initialize a time domain transient simulation or to study the harmonic contents of currents and voltages in the system.

The steady state analysis of power electronics apparatus is usually based on their time domain characteristics [2], [3] considering idealized terminal conditions, such as undistorted terminal voltages or pure dc currents in HVDC systems. The characteristic harmonics of each equipment are determined in this way.

Manuscript received July 20, 2002. This work was supported in part by the Natural Sciences and Engineering Research Council of Canada and in part by CAPES Foundation.

L. T. G. Lima is on leave from TEE/PGMEC, Universidade Federal Fluminense, Niterói, Rio de Janeiro 68501, Brazil. He is now with the Department of Electrical and Computer Engineering, University of Toronto, ON M5S 3G4, Canada (e-mail: leonardo.lima@ieee.org).

A. Semylen, and M. R. Iravani are with the Department of Electrical and Computer Engineering, University of Toronto, ON M5S 3G4, Canada (e-mail: adam.semlyen@utoronto.ca; iravani@ecf.utoronto.ca).

Digital Object Identifier 10.1109/TPWRD.2003.813805
The interaction of these equipment with the network will produce harmonic distortion and, therefore, uncharacteristic harmonics may appear. These harmonics are called uncharacteristic because they do not follow the pattern established in the idealized analysis, with operating conditions seldom observed in practice.

The harmonic distortion is affected by the network components and topology and may vary as the operation conditions change.

Time domain simulation, based on the integration of the differential equations that describe the system, is a time consuming solution when applied to the determination of the periodic steady state [4]-[7].

The use of frequency domain techniques with a Newton-Raphson procedure for the solution of the nonlinear algebraic equations is an efficient approach for the determination of the periodic steady state of power systems containing switched and/or nonlinear components and is the object of this study [4], [6], [7].

This paper presents, in Section II, a generic framework for the frequency domain representation of the periodic steady state of power electronics apparatus. The methodology can be applied to represent line-commutated and forced-commutated equipment and produces analytical models suitable for a Newton-type solution of the harmonic power flow problem. These models can be applied to analyze unbalanced and asymmetric operating conditions, taking fully into account the harmonic distortion of currents and voltages. They are, therefore, able to efficiently handle studies of harmonic interactions.

The use of this methodology is exemplified in the paper by modeling the TCR, as presented in Section III. This is a simple apparatus with only one applied voltage and only one resulting current, but it can be used as a building block for representing an SVC or TCSC, as shown in Section IV.

Frequency domain models for the TCR are available, based on several different techniques such as switching functions [8], FFT transforms [5], or discrete convolution [9]. The proposed model is similarly defined as a voltage controlled current injection in the harmonic domain, but these harmonic components of the current are analytically expressed as functions of the voltage, allowing a straight-forward linearization.

Section V presents a set of results, obtained with a simple test system, to illustrate the main characteristics of the methodology, with emphasis on convergence and robustness of the Newton-type harmonic power flow and the resulting harmonic contents of currents and voltages. 


\section{Harmonic Power Flow Model of POWER EleCtronic APPARATUS}

Power electronics-based apparatus can be modeled in periodic steady state by considering the terminal voltages as inputs and the currents as outputs. This is a convenient choice when a nodal approach is used to represent the power system and yields the separation of the network in its linear and nonlinear parts, allowing a modular implementation of the harmonic power flow.

From the knowledge of the terminal voltages, it is possible to establish the states of conduction of the apparatus in the time domain. These states are defined by the turn-on and turn-off instants of each semiconductor device, considered as an ideal switch. Each state will, thus, be characterized as a time interval and the respective set of conducting valves [10]-[12].

Each of these states corresponds to a linear circuit which can be modeled as a set of linear ordinary differential equations (ODEs) that fully describe the circuit behavior in terms of currents. The analytical solution is readily available and consists of a particular (steady state) solution and a homogeneous (transient) solution. The coefficients of the transient solution and turn-off instants of line-commutated devices are usually not known at this step.

To determine these variables, boundary conditions at each state transition are imposed to ensure the continuity and the periodicity of the solution over the full cycle. This may lead, in a general case, to a set of nonlinear algebraic equations that will require a numerical solution.

The currents are now fully determined as analytical functions at each state (i.e., at each time interval within a cycle). Fourier coefficients are obtained taking the appropriate integrals over a period.

This approach will be illustrated in the next section.

\section{THYRISTOR-CONTROLLED REACTOR (TCR)}

Fig. 1 presents the basic structure of a TCR. The steady state waveforms of a TCR are shown in Fig. 2 [2].

The TCR has noncontinuous current conduction and the turn-on instants $t_{\mathrm{on} 1}$ and $t_{\mathrm{on} 2}$ are defined by the firing signal, obtained from a delay $\alpha$ with respect to a reference (e.g., the zero crossing $t_{0}$ of a signal):

$$
t_{\mathrm{on}}=t_{0}+\alpha
$$

Note that $t_{\mathrm{on} 1}$ and $t_{\mathrm{on} 2}$ can be considered as independent parameters, thus allowing asymmetric firing and, therefore, the presence of even harmonics. This unusual operating condition could arise, for instance, from the use of an SVC to mitigate load unbalance.

In Fig. 2, the reference for the firing delay is, for simplicity, the zero-crossing of the applied voltage. The turn-off instants $t_{\text {off } 1}$ and $t_{\text {off2 }}$ correspond to the zero-crossing instants of the current.

Therefore, the TCR has four states: two on-states and two offstates per cycle. The boundary condition between consecutive states is always associated with current equal to zero.

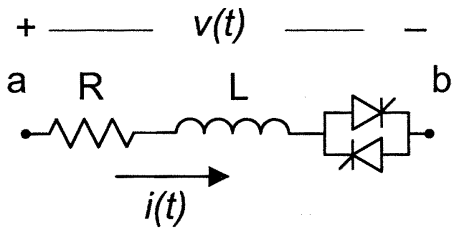

Fig. 1. Thyristor-controlled reactor.

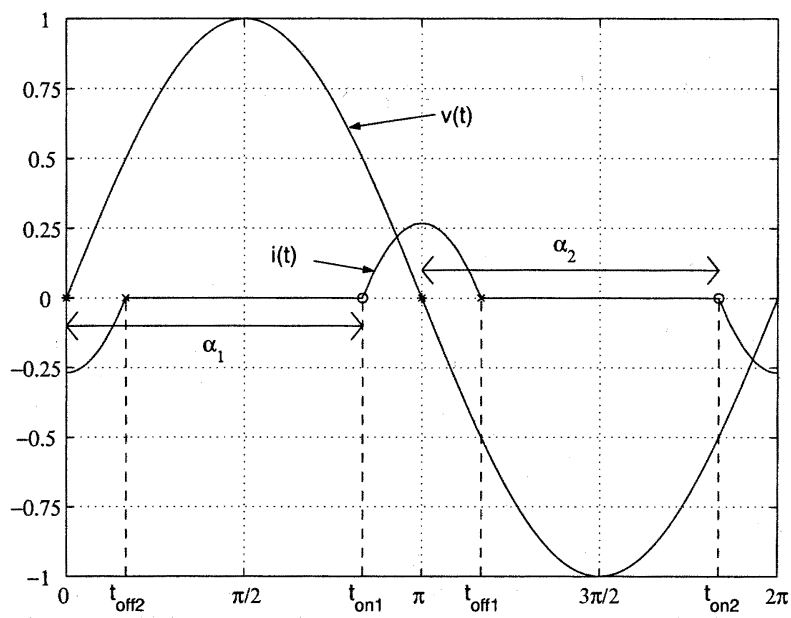

Fig. 2. TCR time domain characteristics.

\section{A. Nonlinear Model}

During thyristor conduction, the following ODE applies:

$$
L \frac{d i}{d t}+R i=v .
$$

The applied voltage is assumed to be known and is expressed in terms of the Fourier coefficients as

$$
v(t)=V_{0}+\sum_{\ell=1}^{n}\left[V_{\ell}^{\prime} \cos (\ell \omega t)+V_{\ell}^{\prime \prime} \sin (\ell \omega t)\right] .
$$

The general solution of (2), considering that the voltage is expressed as in (3), is given by

$$
\begin{aligned}
i(t)= & K e^{-\frac{R}{L}\left(t-t_{\mathrm{on}}\right)}+\frac{V_{0}}{R} \\
& +\sum_{\ell=1}^{n}\left[\frac{V_{\ell}^{\prime}}{Z_{\ell}} \cos \left(\ell \omega t-\varphi_{\ell}\right)+\frac{V_{\ell}^{\prime \prime}}{Z_{\ell}} \sin \left(\ell \omega t-\varphi_{\ell}\right)\right]
\end{aligned}
$$

where

$$
Z_{\ell}=\sqrt{R^{2}+(\ell \omega L)^{2}}
$$

and

$$
\varphi_{\ell}=\tan ^{-1}\left(\frac{\ell \omega L}{R}\right) .
$$

The constant $K$ can be determined from the initial condition $i\left(t_{\mathrm{on}}\right)=0$

$$
\begin{aligned}
K=-\frac{V_{0}}{R}-\sum_{\ell=1}^{n} \frac{V_{\ell}^{\prime}}{Z_{\ell}} \cos \left(\ell \omega t_{\mathrm{on}}-\varphi_{\ell}\right) & \\
& -\sum_{\ell=1}^{n} \frac{V_{\ell}^{\prime \prime}}{Z_{\ell}} \sin \left(\ell \omega t_{\mathrm{on}}-\varphi_{\ell}\right) .
\end{aligned}
$$


Equations (4)-(7) are valid at both conduction periods $t_{\text {on1 }} \leq$ $t \leq t_{\text {off } 1}$ and $t_{\text {on } 2} \leq t \leq t_{\text {off2 }}$ and since the TCR current is periodic, it can be expressed as a Fourier series

$$
i(t)=I_{0}+\sum_{h=1}^{n}\left[I_{h}^{\prime} \cos (h \omega t)+I_{h}^{\prime \prime} \sin (h \omega t)\right] .
$$

The harmonic components of the TCR current shown in (8) can be obtained either numerically, using an FFT algorithm, or analytically.

Appendix A presents the details of the analytical derivation of the Fourier coefficients of the current. The harmonic components of the TCR current are analytical functions of the harmonic components of voltage, turn-on and turn-off instants

$$
i(h)=f\left(v(h), t_{\mathrm{on}}, t_{\mathrm{off}}\right)
$$

where $t_{\text {on }}=\left[\begin{array}{ll}t_{\text {on } 1} & t_{\mathrm{on} 2}\end{array}\right]^{T}, t_{\text {off }}=\left[\begin{array}{ll}t_{\text {off } 1} & t_{\text {off } 2}\end{array}\right]^{T}$ and $i(h)$ and $v(h)$ are harmonic domain vectors. All variables in following sections are in the harmonic domain and, for simplicity, the notation of function of $h$ will be omitted.

The firing of the thyristors determines $t_{\mathrm{on}}$, as shown in (1), based on the zero-crossing of a reference voltage and a firing delay. The zero-crossing of the voltage is numerically obtained by a Newton algorithm applied to the corresponding function, in the form of (3).

To obtain $t_{\text {off }}$, a Newton procedure is applied to (4). The derivative of the current is given by (2) and it is known since at $t=t_{\text {off }}$, the current is zero (by definition). A modified Newton procedure based on the knowledge of the derivative of the function at the solution point is shown in Appendix B. This procedure is proven to have cubic convergence and is also more robust than the conventional Newton algorithm.

\section{B. Linearized Model}

The linearization of (9) can be expressed as

$$
\Delta i=S_{v} \Delta v+s_{t_{\mathrm{on}}} \Delta t_{\mathrm{on}}+s_{t_{\mathrm{off}}} \Delta t_{\mathrm{off}}
$$

where the sensitivity matrix $S_{v}$ and the sensitivity vectors $s_{t_{\text {on }}}$ and $s_{t_{\text {off }}}$ are obtained as partial derivatives of (9) with respect to $v, t_{\mathrm{on}}$, and $t_{\text {off }}$, respectively.

As proved in Appendix $\mathrm{C}$, the harmonic components of the current do not depend on incremental changes in $t_{\text {off }}$ so that $s_{t_{\text {off }}}$ is zero. The Jacobian matrix $S_{v}$ and the column vector $s_{t_{\text {on }}}$ can be accurately obtained from the analytical functions presented in Appendix A.

The delay $\alpha$ is usually the output of a closed-loop control system, controlling voltages and/or currents in the system. The zero-crossing of the reference voltage (or current) changes due to variations in the voltage harmonic contents. The sensitivity of $t_{0}$ to these changes is shown in Appendix D.

Considering $t_{\mathrm{on}}$ as given in (1), the linearized model for the TCR can be expressed as

$$
\Delta i=\left(S_{v}+\frac{1}{d} s_{t_{\mathrm{on}}} s_{0}^{T}\right) \Delta v+s_{t_{\mathrm{on}}} \Delta \alpha .
$$

Equation (11) represents the linearized model for the TCR and the increments in the harmonic components of the current are expressed as a function of the increments in the harmonic components of the voltage and in the firing delay $\alpha$. A harmonic admittance matrix can be defined for this model as

$$
Y_{\mathrm{TCR}}=S_{v}+\frac{1}{d} s_{t_{\mathrm{on}}} s_{0}^{T}
$$

It comprises a primitive sensitivity matrix $S_{v}$, corresponding to the direct effect in the current produced by changes in the voltage, and a rank one contribution obtained as a column vector by row vector multiplication $(1 / d) s_{t_{\mathrm{on}}} s_{0}^{T}$, related to the changes in $t_{\mathrm{on}}$.

\section{POWER SYSTEM MODEL}

The modular approach to the harmonic power flow is based on the partition of the system in its linear part (LP) and nonlinear part (NLP) [13], [3]. The TCR branch described in the previous section can be used as the building block for two different nonlinear apparatus: the SVC and the TCSC.

\section{A. Static VAr Compensator (SVC)}

A typical configuration of the SVC (six-pulse) consists of three delta-connected TCR branches in parallel with grounded $Y$ fixed or thyristor-switched capacitor banks. A 12-pulse configuration is obtained similarly to the HVDC converter, with parallel $Y / Y$ and $Y / \Delta$ connected transformers providing a $30^{\circ}$ phase-shift between two six-pulse SVCs.

The firing control is basically associated with the voltage regulation function, with the firing delay $\alpha$ being adjusted to ensure adequate voltage support by changing the net reactive power injection of the SVC.

\section{B. Thyristor-Controlled Series Capacitor (TCSC)}

The basic configuration of the TCSC has a series capacitor shunted by a TCR branch. The TCSC acts like a tunable parallel LC circuit in series with the line.

The steady-state basic function of the TCSC is active power flow control and it mostly operates within the capacitive range.

\section{Linear Network}

The linear part (LP) of the power system network is represented by its admittance matrix $Y_{h}$ obtained at each harmonic frequency $h \omega$. This approach is simple and powerful, allowing the use of lumped and distributed parameter models and a direct and accurate representation of frequency-dependent parameters.

\section{Harmonic Power Flow}

The harmonic power flow is based on the solution of

$$
Y v=i
$$

where $Y$ is a block diagonal matrix built from the nodal admittance matrices associated with the LP of the system, calculated at frequency $h \omega$. Vector $v$ is the vector of nodal voltages (phasors) at the considered frequencies and $i$ is the vector of nodal current injections (also phasors) from the nonlinear components and the independent sources.

Each TCR branch injects harmonic currents at two network nodes ( $a$ and $b$ in Fig. 1), with opposite signs. Therefore, the 


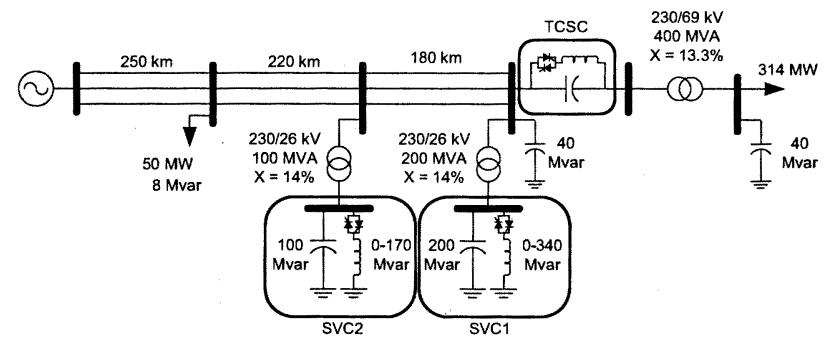

Fig. 3. Example system.

TCR harmonic currents calculated in (9) should be added to the vector $i$ at the proper positions. Moreover, these currents are function of the applied voltage, which can be obtained from the nodal voltages $v$. Therefore, the injected currents $i$ are function of the nodal voltages $i=i(v)$.

The Newton method is applied to solve the following matrix equation:

$$
f(v)=Y v-i(v)=0
$$

and the associated iterative process can be written as

$$
\left.\left[Y-\frac{\partial i}{\partial v}\right]\right|_{v_{k}} \Delta v=-f\left(v_{k}\right)
$$

where the Jacobian matrix is obtained from the linearized models as shown in (12).

\section{RESULTS}

This section presents results intended to highlight key features of the proposed model and its application in a Newton-type harmonic power flow program. The focus is on convergence, computational time, and robustness rather than the operational aspects of the system.

A weak radial system, originally proposed in [14] and also analyzed in [15], is adapted to include a TCSC as shown in Fig. 3. The system data are presented in Appendix E.

The SVCs are originally designed to keep the voltages along the $230-\mathrm{kV}$ transmission system within their normal range of operation. This is a necessary and important task, especially in a weak system where the voltage profile can significantly change with the load variations.

This example system is used to emphasize harmonic distortion in currents and voltages. The choice of the grounded $Y / Y$ connection for the transformers and the lack of harmonic filters are intentional.

The first case is studied with only SVC1 connected, but operating with an odd firing control: each TCR branch has a different delay $\left(110^{\circ}, 120^{\circ}\right.$, and $\left.130^{\circ}\right)$ and there is also a $1^{\circ}$ difference between the positive and the negative half-cycle firings. This firing scheme results in noncharacteristic harmonic generation, with the presence of even harmonics and third harmonic (and multiples) despite the delta connection of the TCR branches. The harmonic contents of phase-to-ground voltages (phases A, $\mathrm{B}$, and C) at the 26-kV terminal bus of SVC1 are shown in Fig. 4.

The Newton method is very robust and accurate, yielding fast convergence to very strict tolerance $\left(\varepsilon \leq 10^{-9}\right)$, as shown in Fig. 5. The current injection and the Norton equivalent methods

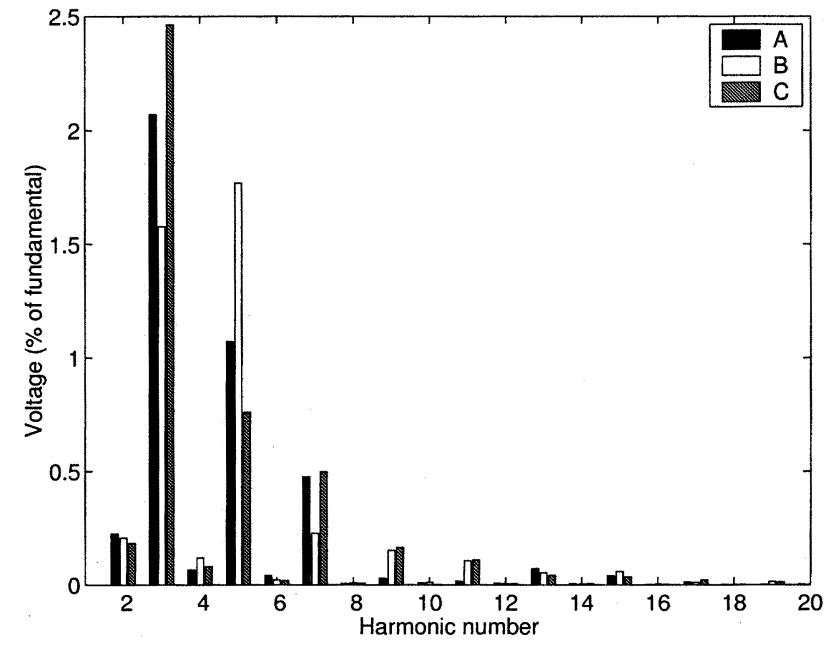

Fig. 4. Case 1-Harmonics of voltage at SVC1 terminal buses.

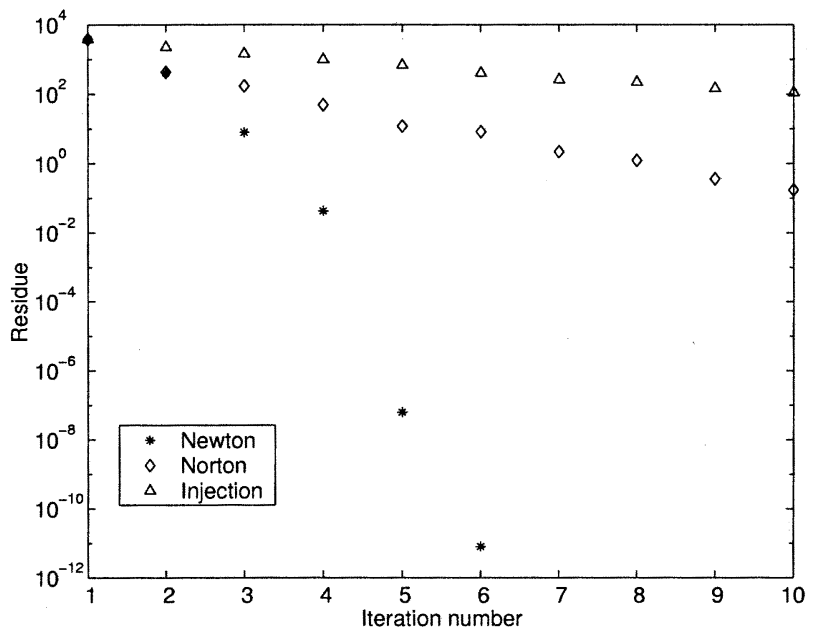

Fig. 5. Harmonic power flow-convergence.

[3], [7] took 32 and 96 iterations, respectively, to reach the same tolerance, considering 50 harmonics.

Fig. 6 presents the average iteration time for these solution methods. The current injection and Norton equivalent methods have almost the same computational cost per iteration and a linear relationship with respect to the number of harmonics considered. The Newton method is more expensive per iteration than the other two methods and its average iteration time has a nonlinear relationship (almost quadratic) with respect to the number of harmonics. All methods calculate the nonlinear injected currents, but the Newton method has the overhead of calculating the Jacobian matrix. Also, the Newton method solves for all frequencies at the same time, with a single matrix of order $n_{\text {bus }} \times n_{h}$, while the current injection and Norton equivalent methods are frequency-decoupled, solving $n_{h}$ problems of order $n_{\text {bus }}$, where $n_{\text {bus }}$ is the number of nodes in the network and $n_{h}$ is the number of harmonics considered in the problem.

On the other hand, the Newton method produced the fastest overall solution due to its small number of iterations (less than six iterations) and the Norton equivalent method will become a good option only if more than 80 harmonics are to be considered. With 50 harmonics, the Newton method required $33.4 \mathrm{~s}$ to 


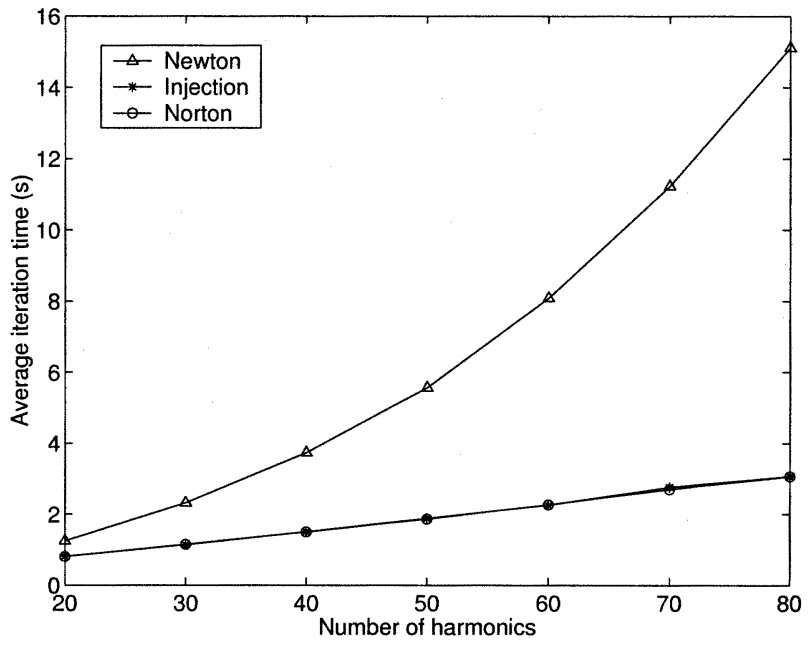

Fig. 6. Harmonic power flow-iteration time.

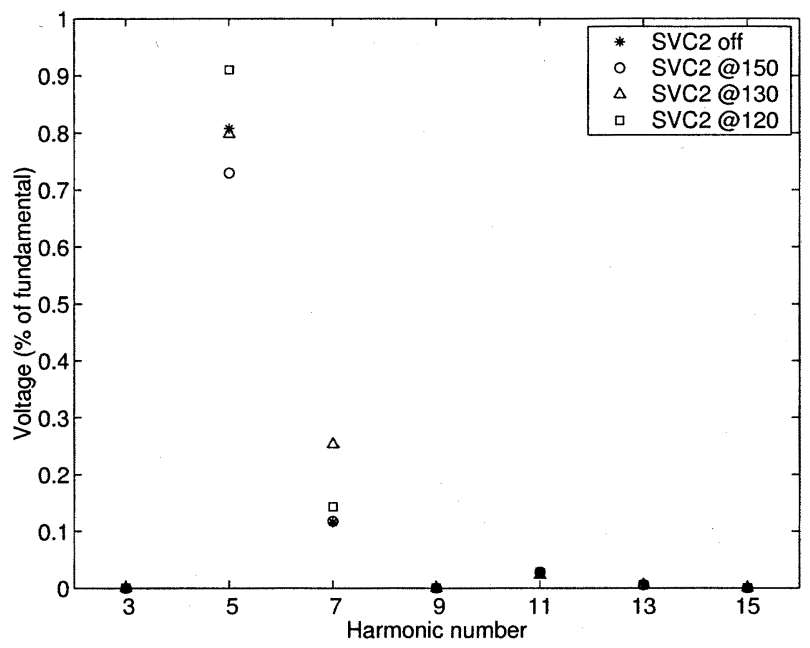

Fig. 7. Harmonic interaction-Two SVCs.

reach convergence, while the Norton equivalent and the current injection methods took $59.6 \mathrm{~s}$ and $180.8 \mathrm{~s}$, respectively.

These results were obtained based on Matlab ${ }^{\circledR}$ implementations of the methods. Different implementations are expected to yield similar conclusions, with the Newton method still resulting in better overall performance for a not too large number of harmonics.

The second study case, with SVC1 and SVC2 connected, is used to illustrate the interaction between two harmonic sources. Fig. 7 presents the harmonic content at the high-voltage terminal of SVC1. The firing angle of SVC1 is kept constant at $\alpha=120^{\circ}$ and both SVCs have balanced and symmetrical firing strategies. The possible presence of a third harmonic component was considered during the iterations but converged to a negligible value at the solution, as expected.

The presence of SVC2 has an impact on the harmonic content of the voltages along the system. Although the absolute change is small at the high-voltage terminal of SVC1, the recommended maximum individual voltage distortion, at this voltage level, is only 1\% [16]. Therefore, an accurate assessment of the harmonic distortion, as provided by this model, is required to ensure compliance with the standards.

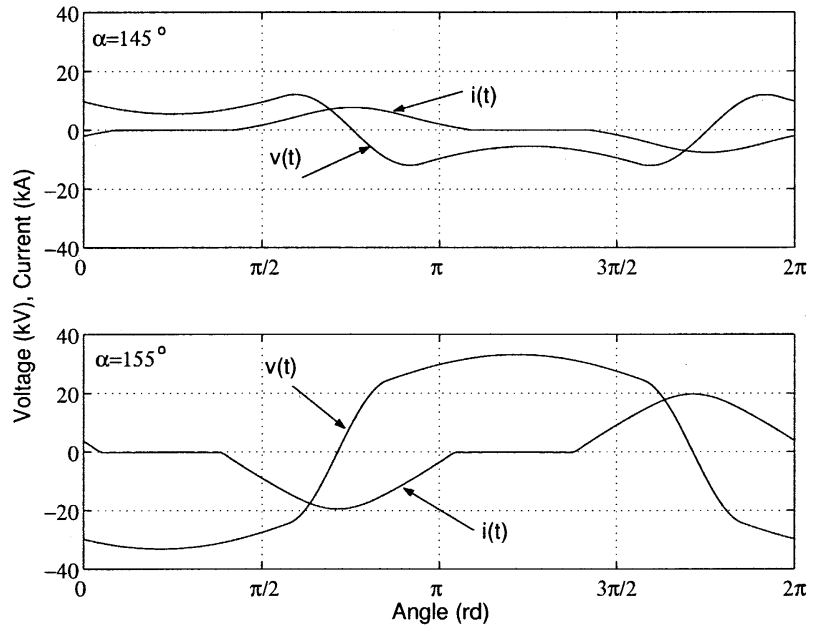

Fig. 8. TCSC voltages and currents.

The third case study considers only the TCSC to be connected. Fig. 8 shows the waveforms of the TCR current and voltage when the TCSC is operating at the capacitive ( $\alpha=$ $\left.155^{\circ}\right)$ and inductive $\left(\alpha=145^{\circ}\right)$ regions, respectively. These waveforms were obtained through an inverse Fourier transform applied to the harmonic domain solution considering contributions up to the 50th harmonic. These waveforms are quite similar to those shown in textbooks like [2].

The first harmonic parallel resonance of the TCSC occurs at around $\alpha=151^{\circ}$ and the Newton method converged without any difficulty to a solution with $\alpha=148^{\circ}$ and with $\alpha=155^{\circ}$. The current injection and the Norton equivalent methods do not converge even after 100 iterations.

\section{CONCLUSION}

This paper presents an analytical model in the harmonic domain to obtain the steady state behavior of a TCR, used as a building block to represent SVCs and TCSCs in a modular harmonic power flow program based on the Newton-Raphson method. The Newton-Raphson approach is arguably the most efficient method for harmonic power flow solution [7] and, therefore, the proposed model is intrinsically associated with that solution method. Although the model can be used in conjunction with injection methods, its major feature is the analytical Jacobian described in Section III.B, necessary to an efficient Newton-Raphson implementation.

The model was developed according to a general framework for the frequency domain representation of the steady state of power electronics apparatus and is considered as the first application and the validation example of this methodology.

The Newton method allows fast, accurate and robust convergence and although it requires simultaneous solution for all considered frequencies, convergence is usually achieved in few iterations, with an overall performance superior to fixed-point iteration methods.

The number of frequencies to be considered is user-defined and corresponds to a truncation of the Fourier series associated with the time-domain steady-state solution. Despite this truncation, the Newton method, as it is formulated in the frequency 
domain considering only the specified frequencies, allows accurate and ultimately quadratic convergence to the truncated solution.

\section{APPENDIX A \\ HARMONIC CURRENT CALCULATION}

The Fourier coefficients in (8) can be obtained from

$$
\begin{aligned}
& I_{h}^{\prime}=\frac{\omega}{\pi} \sum_{j=1}^{2} \int_{t_{\mathrm{on}_{j}}}^{t_{\mathrm{off}_{j}}} i(t) \cos (h \omega t) d t \\
& I_{h}^{\prime \prime}=\frac{\omega}{\pi} \sum_{j=1}^{2} \int_{t_{\mathrm{on}_{j}}}^{t_{\mathrm{off}_{j}}} i(t) \sin (h \omega t) d t
\end{aligned}
$$

and

$$
I_{0}=\frac{\omega}{2 \pi} \sum_{j=1}^{2} \int_{t_{\mathrm{on}_{j}}}^{t_{\mathrm{off}_{j}}} i(t) d t .
$$

In general, these integrals have to be evaluated over a period. The limits of the integrals in (16)-(18) reflect the fact that the current is zero during nonconduction periods. It should be noted that (4) is composed of only four basic function types: constant, exponential, sine, and cosine. Therefore, only a few simple integrals should be calculated. As an example, one of these simpler terms to be determined in (16) is

$$
F_{1}^{\ell, h}=\frac{\omega}{\pi} \sum_{j=1}^{2} \int_{t_{\mathrm{on}_{j}}}^{t_{\mathrm{off}_{j}}} \frac{V_{\ell}^{\prime}}{Z_{\ell}} \cos \left(\ell \omega t-\varphi_{\ell}\right) \cos (h \omega t) d t
$$

and its solution is readily available from tables or symbolic math software. This term is related to a contribution of the harmonic $\ell$ of the voltage to the harmonic $h$ of the current. The term $F_{1}^{\ell, h}$ is a function of the harmonic components of the voltage and the switching instants $t_{\text {on }}$ and $t_{\text {off }}$.

Table A.I presents all of the functions needed to evaluate (16)-(18). They are defined in a similar way as in (19) with the terms indicated in the table.

Using these functions, (16)-(18) can be written as

$$
\begin{aligned}
I_{h}^{\prime}= & F_{14}^{K, h}+F_{2}^{h, h} \\
& +\sum_{\substack{\ell=1 \\
\ell \neq h}}^{n} F_{1}^{\ell, h}+F_{4}^{h, h}+\sum_{\substack{\ell=1 \\
\ell \neq h}}^{n} F_{3}^{\ell, h}+F_{9}^{e, h} \\
I_{h}^{\prime \prime}= & F_{15}^{K, h}+F_{6}^{h, h} \\
& +\sum_{\substack{\ell=1 \\
\ell \neq h}}^{n} F_{5}^{\ell, h}+F_{8}^{h, h}+\sum_{\substack{\ell=1 \\
\ell \neq h}}^{n} F_{7}^{\ell, h}+F_{10}^{e, h}
\end{aligned}
$$

and

$$
I_{0}=F_{16}^{K, 0}+\sum_{\ell=1}^{n} F_{12}^{\ell, 0}+\sum_{\ell=1}^{n} F_{13}^{\ell, 0}+F_{11}^{e, 0}
$$

TABLE A.I

BASIC FUNCTIONS FOR HARMONIC CURRENTS

\begin{tabular}{l|l|l}
\hline Function & Term of $i(t)$ & Fourier Factor \\
\hline$F_{1}^{\ell, h}$ & $\cos \left(\ell \omega t-\varphi_{\ell}\right)$ & $\cos (h \omega t)$ \\
\hline$F_{2}^{h, h}$ & $\cos \left(h \omega t-\varphi_{h}\right)$ & $\cos (h \omega t)$ \\
\hline$F_{3}^{\ell, h}$ & $\sin \left(\ell \omega t-\varphi_{\ell}\right)$ & $\cos (h \omega t)$ \\
\hline$F_{4}^{h, h}$ & $\sin \left(h \omega t-\varphi_{h}\right)$ & $\cos (h \omega t)$ \\
\hline$F_{5}^{\ell, h}$ & $\cos \left(\ell \omega t-\varphi_{\ell}\right)$ & $\sin (h \omega t)$ \\
\hline$F_{6}^{h, h}$ & $\cos \left(h \omega t-\varphi_{h}\right)$ & $\sin (h \omega t)$ \\
\hline$F_{7}^{\ell, h}$ & $\sin \left(\ell \omega t-\varphi_{\ell}\right)$ & $\sin (h \omega t)$ \\
\hline$F_{8}^{h, h}$ & $\sin \left(h \omega t-\varphi_{h}\right)$ & $\sin (h \omega t)$ \\
\hline$F_{9}^{e, h}$ & $e^{-\frac{R}{L}\left(t-t_{0}\right)}$ & $\cos (h \omega t)$ \\
\hline$F_{10}^{e, h}$ & $e^{-\frac{R}{L}\left(t-t_{0}\right)}$ & $\sin (h \omega t)$ \\
\hline$F_{11}^{e, 0}$ & $e^{-\frac{R}{L}\left(t-t_{0}\right)}$ & 1 \\
\hline$F_{12}^{\ell, 0}$ & $\cos \left(\ell \omega t-\varphi_{\ell}\right)$ & 1 \\
\hline$F_{13}^{\ell, 0}$ & $\sin \left(\ell \omega t-\varphi_{\ell}\right)$ & 1 \\
\hline$F_{14}^{K, h}$ & $K$ & $\cos (h \omega t)$ \\
\hline$F_{15}^{K, h}$ & $K$ & $\sin (h \omega t)$ \\
\hline$F_{16}^{K, 0}$ & $K$ & 1 \\
\hline
\end{tabular}

APPENDIX B

Modified NEwTON PROCEDURE

Consider a nonlinear function with a root at the origin $(f(0)=0)$ and expressed as a truncated Taylor expansion around the solution point $x=0$ :

$$
f(x)=x+a x^{2}+2 \theta x^{3} .
$$

If the derivative of this function at the solution point is known, a modified Newton procedure (MNP) defined as

$$
x_{k+1}=x_{k}-\frac{f\left(x_{k}\right)}{f_{\text {ave }}^{\prime}}
$$

where

$$
f_{\mathrm{ave}}^{\prime}=\frac{f^{\prime}\left(x_{k}\right)+f^{\prime}(0)}{2}
$$

results in a faster (ultimately cubic convergence) and a more robust iterative procedure.

Combining the expressions above, we get

$$
x_{k+1}=x_{k}-\frac{x_{k}+a x_{k}^{2}+2 \theta x_{k}^{3}}{1+a x_{k}+3 \theta x_{k}^{2}}
$$

and, as $x_{k}$ converges to the solution $(x=0)$, this expression reduces to

$$
x_{k+1} \approx \theta x_{k}^{3}
$$


The robustness of MNP can be assessed in a sufficiently small neighborhood of the solution at the origin by using the quadratic approximation

$$
f(x)=x+a x^{2}
$$

of (23). Its extremum is at

$$
x_{0}=-\frac{1}{2 a} \text {. }
$$

For conventional Newton iterations, $x_{0}$ represents a one-sided boundary where the tangent to the parabola (28) becomes parallel to the $x$-axis. No such restriction applies however to MNP. The approximation (28) corresponds to (23) if $\theta \rightarrow 0$, and therefore, by (27), $x_{k+1} \rightarrow 0$. This means that the solution is reached in a single step from any $x_{k}$ which is not too far away from the solution for approximation (28) to be valid. Thus, $x_{0}$ of (29) does not constitute a boundary for $x_{k}$ for the convergence of MNP.

\section{APPENDIX C SENSITIVITY TO $t_{\text {off }}$}

The harmonics of the TCR current $i(t)$ are given by the Fourier coefficients calculated as

$$
I_{h}=K \int_{t_{\mathrm{on}}}^{t_{\mathrm{off}}} i(t) f_{h}(t) d t
$$

where $K$ is a constant and $f_{h}(t)$ is a sine or cosine function of $h \omega t$ (or 1 for the dc component).

The sensitivity of $I_{h}$ to changes in $t_{\text {off }}$ is given by the integrand in (30) at its upper limit $t_{\mathrm{off}}$

$$
\frac{\partial I_{h}}{\partial t_{\text {off }}}=K i\left(t_{\text {off }}\right) f_{h}\left(t_{\text {off }}\right) .
$$

Since $i\left(t_{\mathrm{off}}\right)=0$, it follows that:

$$
\frac{\partial I_{h}}{\partial t_{\text {off }}}=0
$$

and therefore $s_{t_{\text {off }}}=0$.

Note, however, that $i(t)$ is a function of $t_{\text {on }}$ and we cannot conclude that the sensitivity of $I_{h}$ to changes in $t_{\text {on }}$ is also zero.

\section{APPENDIX D \\ SENSITIVITY OF $t_{\text {on }}$}

Given a voltage, expressed in its harmonic components as (3), its zero crossing occurs at $v\left(t_{0}\right)=0$. The total derivative of this expression, calculated at $t=t_{0}$, is given by

$$
\begin{aligned}
\Delta V_{0} & +\sum_{h=1}^{n_{h}} \cos \left(h \omega t_{0}\right) \Delta V_{h}^{\prime}+\sum_{h=1}^{n_{h}} \sin \left(h \omega t_{0}\right) \Delta V_{h}^{\prime \prime} \\
& -\sum_{h=1}^{n_{h}} h \omega\left[V_{h}^{\prime} \sin \left(h \omega t_{0}\right)-V_{h}^{\prime \prime} \cos \left(h \omega t_{0}\right)\right] \Delta t_{0}=0 .
\end{aligned}
$$

The expression for $\Delta t_{0}$ can be written, in vector form, as

$$
\Delta t_{0}=\frac{1}{d}\left[s_{0}^{T} \Delta v\right]
$$

where $s_{0}^{T}$ is a row vector, $\Delta v$ is the vector containing all the harmonic components $\Delta V_{0}, \Delta V_{h}^{\prime}$, and $\Delta V_{h}^{\prime \prime}$. The scalar $d$ is the derivative of $v(t)$ calculated at $t_{0}$.

If the zero-crossing of some other variable-say, $i_{\text {line }}(t)$ of a TCSC, rather than $v(t)$-is used as a reference, we first express $i_{\text {line }}(t)$ as a function of voltage, and therefore, expressed in a Fourier form as (3). Then, (34) remains formally unchanged and yields directly $\Delta t_{0}$ for the zero crossing of $i_{\text {line }}(t)$. The performance of the model is thus unaffected by the particular choice of a reference.

\section{APPENDIX E EXAMPLE SYSTEM DATA}

Only positive sequence data were available [14], [15] and some simplifying assumptions were made to obtain a full threephase model.

- Transmission lines are considered to be transposed and are represented as coupled three-phase $\pi$ sections with $R, L$ and $C$ matrices calculated at $60 \mathrm{~Hz}$. These matrices are considered, for simplicity, to be constants at all harmonic frequencies. All circuits have the same parameters

$$
\begin{aligned}
& r_{1}=0.09 \Omega / \mathrm{km} \quad x_{1}=0.5 \Omega / \mathrm{km} \\
& y_{1}=3.28 \times 10^{-6} \mathrm{~S} / \mathrm{km} \\
& r_{0}=0.44 \Omega / \mathrm{km} \quad x_{0}=1.7 \Omega / \mathrm{km} \\
& y_{0}=2.16 \times 10^{-6} \mathrm{~S} / \mathrm{km} .
\end{aligned}
$$

- Transformers are modeled as three-phase banks of singlephase units connected in grounded $Y / Y$, with the leakage inductance calculated from the per unit values shown in Fig. 3 at $60 \mathrm{~Hz}$.

- The loads are considered to be constant resistances and inductances, calculated at $60 \mathrm{~Hz}$.

- The TCSC, when present, has a capacitance that compensates $40 \%$ of the equivalent $60-\mathrm{Hz}$ reactance of the three parallel circuits at the last section $(180 \mathrm{~km})$. The TCR branch has a full conduction inductive reactance of $10 \%$ of the capacitive reactance.

\section{ACKNOWLEDGMENT}

The first author wishes to thank Universidade Federal Fluminense for his leave of absence.

\section{REFERENCES}

[1] Task Force, "Guidelines for modeling power electronics in electric power engineering applications," IEEE Trans. Power Delivery, vol. 12, no. 1, pp. 505-514, Jan. 1997. Task Force on Power Electronics Modeling.

[2] N. G. Hingorani and L. Gyugyi, Understanding FACTS-Concepts and Technology of Flexible AC Transmission Systems. New York, NY: IEEE Press, 2000.

[3] J. Arrillaga, B. C. Smith, N. R. Watson, and A. R. Wood, Power System Harmonic Analysis. New York: Wiley, 1997.

[4] A. Semlyen, E. Acha, and J. Arrillaga, "Newton-type algorithms for the harmonic phasor analysis of nonlinear power circuits in periodical steady state with special reference to magnetic nonlinearities," IEEE Trans. Power Delivery, vol. 3, pp. 1090-1098, July 1988.

[5] W. Xu, J. R. Marti, and H. W. Dommel, "A multiphase harmonic load flow solution technique," IEEE Trans. Power Syst., vol. 6, pp. 174-182, Feb. 1991. 
[6] E. Acha and M. Madrigal, Power Systems Harmonics: Computer Modeling and Analysis. New York: Wiley, 2001.

[7] L. T. G. Lima, A. Semlyen, and M. R. Iravani, "Fast initialization for transient calculations with nonsinusoidal steady state," in Proc. Int Power Syst. Transients Conf., June 2001, pp. 369-374.

[8] L. J. Bohmann and R. H. Lasseter, "Harmonic interactions in thyristor controlled reactor circuits," IEEE Trans. Power Delivery, vol. 4, pp. 1919-1926, July 1989.

[9] J. J. Rico, E. Acha, and T. J. E. Miller, "Harmonic domain modeling of three phase tyristor-controlled reactors by means of switching vectors and discrete convolutions," IEEE Trans. Power Delivery, vol. 11, pp. 1678-1684, July 1996.

[10] R. H. Lasseter, S. G. Jalali, and I. Dobson, "Dynamic response of a thyristor controlled switched capacitor," IEEE Trans. Power Delivery, vol. 9, pp. 1609-1615, July 1994.

[11] B. K. Perkins and M. R. Iravani, "Novel calculation of hvdc converter harmonics by linearization in the time-domain," IEEE Trans. Power Delivery, vol. 12, pp. 867-873, Apr. 1997.

[12] - "Dynamic modeling of a tcsc with application to ssr analysis," IEEE Trans. Power Syst., vol. 12, pp. 1619-1625, Nov. 1997.

[13] A. Semlyen and M. Shlash, "Principles of modular harmonic power flow methodology," in Proc. Inst. Elect. Eng._Gen., Transm. Dist., vol. 147, Jan. 2000, pp. 1-6.

[14] A. J. P. Ramos and H. Tyll, "Dynamic performance of a radial weak power system with multiple static var compensators," IEEE Trans. Power Syst., vol. 4, pp. 1316-1325, Nov. 1989.

[15] M. Parniani and M. R. Iravani, "Computer analysis of small-signal stability of power systems including network dynamics," in Proc. Inst. Elect. Eng. -Gen., Transm. Dist., vol. 142, Nov. 1995, pp. 613-617.

[16] IEEE Recommended Practices and Requirements for Harmonic Control in Electrical Power Systems (ANSI), 1992.
Leonardo T. G. Lima (M'90) received the B.Sc., M.Sc., and D.Sc. degrees from Federal University, Rio de Janeiro, Brazil, in 1986, 1991, and 1999, respectively.

Currently, he is an Associate Professor in the Electrical Engineering Department at Universidade Federal Fluminense, Niterói, Rio de Janeiro, Brazil, an is with the Department of Electrical and Computer Engineering at the University of Toronto, ON, Canada, on a post-doctoral leave of absence. His interest include power systems dynamics and control, harmonics, and electromagnetic transient analysis.

Adam Semlyen (LF'97) was born in 1923 in Rumania. He received the Dipl.Ing. degree and the Ph.D. from Polytechnic Institute of Timisoara, Iasi, Romania.

Currently, he is a Professor in the Department of Electrical and Computer Engineering at the University of Toronto, ON, Canada. He began his career at the University of Toronto with an electric power utility and held academic positions at the Polytechnic Institute of Timisoara. His research interests include steady state and dynamic analysis as well as computation of electromagnetic transients in power systems.

M. R. Iravani (F'03) received the B.Sc. degree in electrical engineering from Tehran Polytechnique University, Iran, in 1976, and received the M.Sc. and $\mathrm{Ph} . \mathrm{D}$. degrees in electrical engineering from the University of Manitoba, Winnipeg, MB, Canada, in 1981 and 1985, respectively.

Currently, he is a Professor at the University of Toronto, ON, Canada. His research interests include power electronics and power system dynamics and control 